\title{
Effect of Buteyko Breathing Technique on Asthma Severity Control among School Age Children
}

Esraa Mohammed, Fawzia Abusaad and Boshra Attia

Pediatric Nursing, Faculty of Nursing Mansoura University, Egypt

\section{III}

Background: Asthma is a complex condition that can impair the child's physical growth. It affect not only the child's physical status but also his optimal functional capacity and performance. Buteyko Breathing Technique is an exercise designed to regulate the breathing process. Aim: This study aimed to study the effect of Buteyko Breathing Technique (BBT) on asthma severity control among school age children. Materials and Methods: A quasi-experimental design (one group pre and posttest) was conducted on 33 asthmatic school age child who attended the sensitivity outpatient clinics at Mansoura University Children's Hospital (MUCH). Tools: Structured interview sheet for child and their parent, Childhood Asthma Control Test(C-ACT), Peak Expiratory Flow Rate (PEFR) and Control Pause Test (CP) are used to collect data. Results: The mean C-ACT pretest was significantly improved in the posttest with high mean percent of change of posttest than pretest, which was clinically and statically high significant. There was a statistical significant increase in the mean of PEFR and CP at the fourth week than the first one with a high significant mean percent of change. There was a significant positive decrease in the heart rate over the four weeks of follow up with high mean percent of change at fourth week than first one. Conclusion: This study support the effectiveness of BBT in improving respiratory outcome and promoting asthma control among school age children with bronchial asthma.

Keywords: Bronchial asthma; Buteyko breathing technique; Respiratory outcome; School age children

Editor-in-Chief: Prof. M.L. Salem, PhD - Article DOI: 10.21608/jcbr.2021.59903.1135 